\title{
DIGITAL MATERIALS - EVALUATION OF THE POSSIBILITIES OF USING SELECTED HYPERELASTIC MODELS TO DESCRIBE CONSTITUTIVE RELATIONS
}

\author{
J. MAŃKOWSKI ${ }^{*}$ and J. LIPNICKI \\ Institute of Machine Design Fundamentals \\ Warsaw University of Technology \\ Narbutta Str. 84, 02-524 Warsaw, POLAND \\ E-mail: jaroslaw.mankowski@simr.pw.edu.pl
}

\begin{abstract}
The authors tried to identify the parameters of numerical models of digital materials, which are a kind of composite resulting from the manufacture of the product in 3D printers. With the arrangement of several heads of the printer, the new material can result from mixing of materials with radically different properties, during the process of producing single layer of the product. The new material has properties dependent on the base materials properties and their proportions. Digital materials tensile characteristics are often non-linear and qualify to be described by hyperelastic materials models. The identification was conducted based on the results of tensile tests models, its various degrees coefficients of the polynomials to various degrees coefficients of the polynomials. The Drucker's stability criterion was also examined. Fourteen different materials were analyzed.
\end{abstract}

Key words: digital materials, hyperelastic, identification of models, FEM, 3D printing, PolyJet Matrix technology.

\section{Introduction}

The term digital materials, according to the definition proposed in 2007 by Popescu MIT [1], refers to materials made of different components that meet the following criteria: "The set of all the components used in a digital material is finite (i.e., discrete parts). The set of the all joints the components of a digital material can form is finite (i.e., discrete joints). The assembly process has complete control over the placement of each component ( i.e., explicit placement).". The wording refers, in fact, to the properties of the finally formed structure and is closely linked to the way of production. In two of the latest 3D printing technologies: PolyJet Matrix and Triple-Jet final material properties are also achieved during the manufacture of the finished product. The base materials are liquid polymer resins cured with UV light [2]. The resultant product is a kind of a composite formed directly in the manufacturing process. The arrangement of multiple printheads, "shot-firing" two or three components in a strictly limited way (a precisely defined droplet base materials) form a mixture and produce a single layer of a perpetuated material, whose properties are different than the base materials. Therefore, it seems justified to use the term "digital materials" which appears both in the manufacturer's information [3] and more frequently in the conference and scientific publications describing manufacturing subjects [4] and studies on the properties of digital materials $[5,6]$. 3D printers that use these technologies render it possible to process several materials at the same time in a single printing process which allows the creation of structures whose cross-sections have variable physical properties.

In the case of materials, which were tested for this publication, attention should be driven to the fact that the tested materials were created by mixing two components with diametrically different properties. One of them is a relatively rigid material, the other is a "rubber-like" material. Both materials have non-linear

\footnotetext{
${ }^{*}$ To whom correspondence should be addressed
} 
characteristics of stretch. The resulting combination of materials has intermediate properties, and the tensile characteristics allow defining it as hyperelastic.

Due to the fact that these materials are relatively new, and there are few publications containing data that can be used in computational models, the authors tried to identify the constitutive models for fourteen of them. Thanks to the use of the results of Łąpieś et al. presented at the conference "Methods \& Tools for CAE - Concepts and Applications" [7], material models were identified.

\section{The object of research}

Fourteen different digital materials, obtained by the combination of various photopolymer resins were examined. Two sets of materials named "Tango" and "Vero" were analyzed. The first, is characterized by non-linear elasticity and the ability to achieve high elastic deformations; and the other, also has non-linear characteristics, but is much stiffer and more durable [8]. Figure 1 shows the characteristics obtained during the static uniaxial tension tests. Tests were carried out for dog-bone specimens, made in accordance with EN ISO 527-2 [9]. The dimensions of the samples are shown in Fig.2.
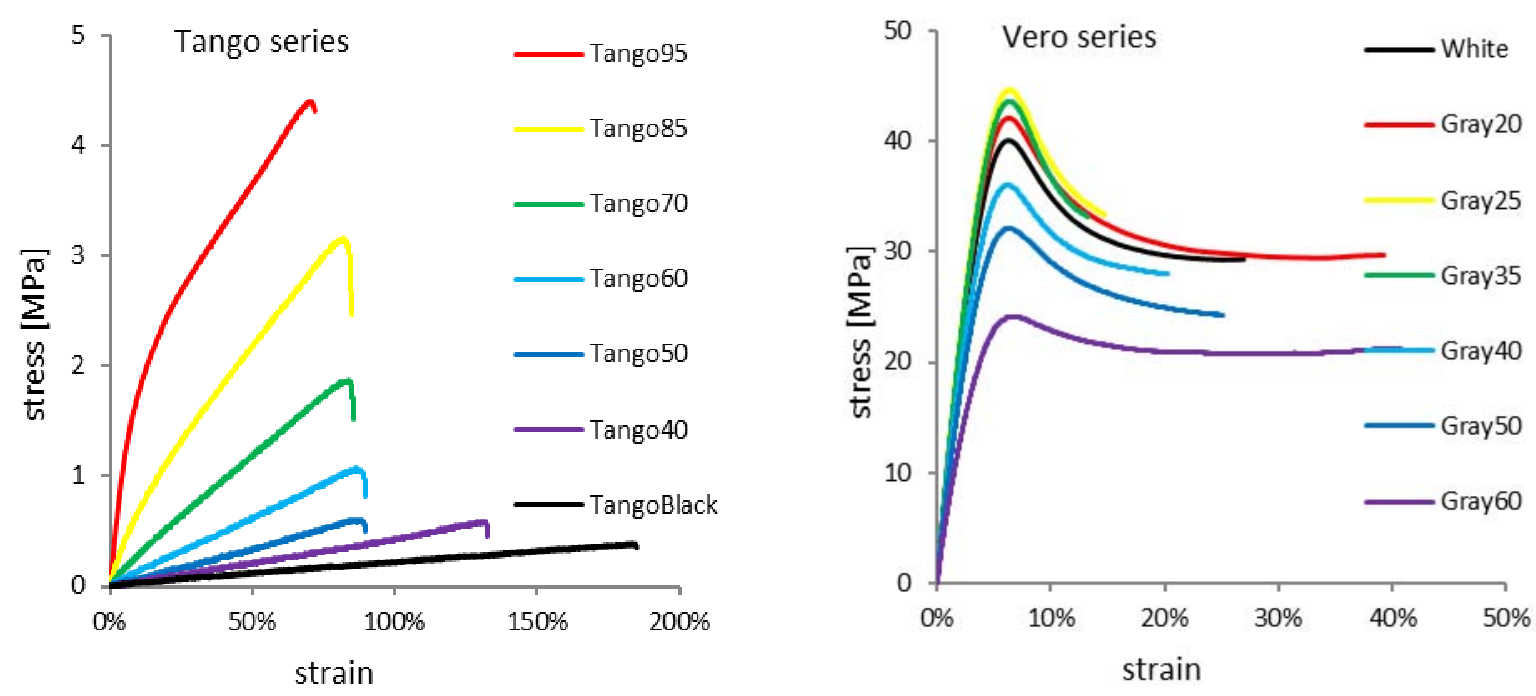

Fig.1. Tensile characteristics of the materials from Tango and Vero groups.

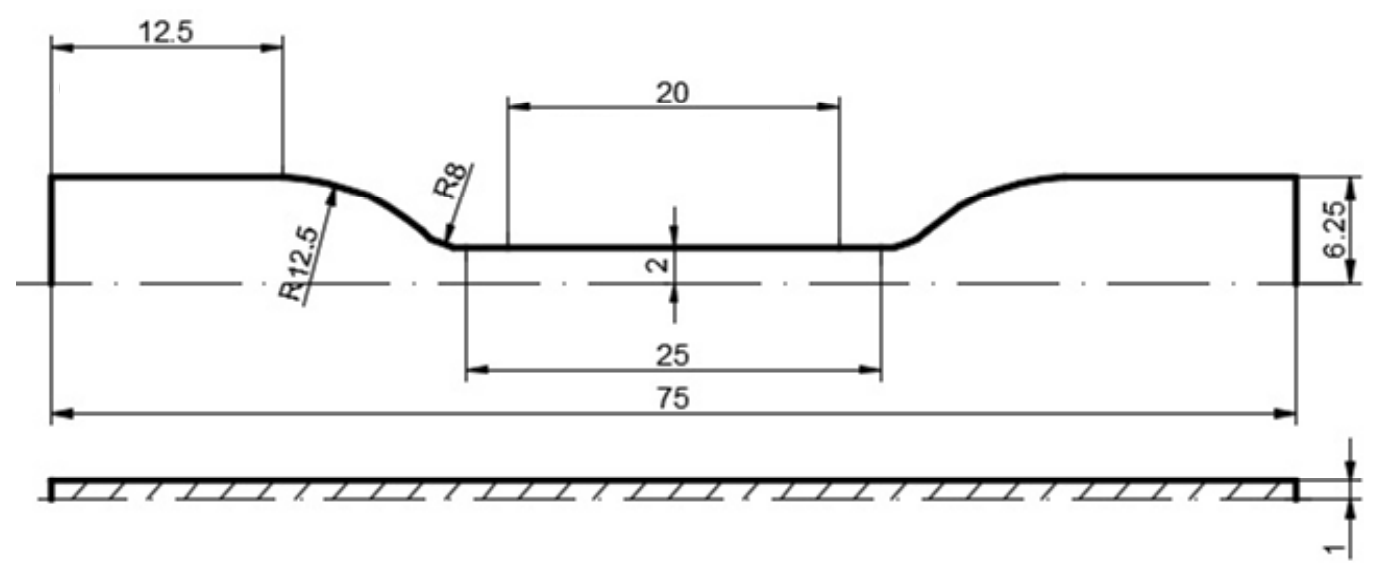

Fig.2. The dimensions of the specimens used for tensile tests. 


\section{The aim of study}

The aim of the work was to evaluate the possibility of using selected models of hyperelastic materials for the description of constitutive compounds of digital materials.

\section{Tested material models}

As already mentioned, the majority of materials examined in this study have hyperelastic characteristics. Therefore, constitutive models can be identified by, e.g., using one of the methods described in the work $[10,11]$.

The first approach is the method by statistical mechanics going into the molecular structure of the material, based on the models of the material structure. On the other hand, it is possible to build a mathematical description of the material behavior, without regard to the construction of the structure. This approach results from continuum mechanics, consisting in determining the strain energy density function, based on the deformation of the material, and on its basis to create a description of the stress-strain relationship.

Focusing on the second method, it can be concluded that the hyperelastic material subjected to strain, contains strain energy which is described by the energy density function W. There are many different models describing the form of the $\mathrm{W}$ function. It may depend on various tensors: the deformation or distortion, which causes obtaining different forms of constitutive equations. The energy density function depends only on the current state of strain, but not on the history of the deformation course [12].

If the tested material is isotropic, then to describe the material properties you can use the $\mathrm{W}$ function, expressed by the strain tensor invariants . It can also be described using the appropriate stretches $\lambda$, being the ratio between the whole length of the element subjected to deformation and its original length. By introducing appropriate stretch for three main directions $\lambda_{1}, \lambda_{2}, \lambda_{3}$, a strain tensor invariants form may be obtained Eq.(4.1) [10], which allows the description of the strain energy using the principal stretches Eq.(4.2). Transforming the function (4.2) in a Taylor series, the general form of the strain energy density function of a non-linear elastic material is obtained Eq.(4.3).

$$
\begin{aligned}
& I_{1}=\lambda_{1}^{2}+\lambda_{2}^{2}+\lambda_{3}^{2}, \\
& I_{2}=\lambda_{1}^{2} \lambda_{2}^{2}+\lambda_{2}^{2} \lambda_{3}^{2}+\lambda_{3}^{2} \lambda_{1}^{2}, \\
& I_{3}=\lambda_{1}^{2} \lambda_{2}^{2} \lambda_{3}^{2}=J^{2}
\end{aligned}
$$

where: $J$ - is the total volumetric ratio.

$$
\begin{aligned}
& W=W\left(\lambda_{1}^{2}, \lambda_{2}^{2}, \lambda_{3}^{2}\right), \\
& W=\sum_{i, j, k=0}^{\infty} C_{i j k}\left(I_{1}-3\right)^{i}\left(I_{2}-3\right)^{j}\left(I_{3}-3\right)^{k} .
\end{aligned}
$$

Because of the material incompressibility, after separating deviatoric and volumetric parts, typical hyperelastic material models usually take the polynomial form Eq.(4.4), where $C_{i j}$ and $D_{k}$ are material constants which have to be determined by tests. 


$$
W=\sum_{i+j=1}^{N} C_{i j}\left(I_{1}-3\right)^{i}\left(I_{2}-3\right)^{j}+\sum_{k=1}^{N} \frac{1}{D_{k}}(J-1)^{2 k}
$$

Equation (4.4), over the years, was modified to less or more complicated forms by introducing a different number of parameters describing the material behavior [13]. The study tested a number of wellknown polynomial models, implemented i.a. in the Abaqus system: Polynominal (4.4), Reduced Polynominal (4.5) and Ogden (4.6), Van der Waals (4.7).

$$
\begin{aligned}
& W=\sum_{i=1}^{N} C_{i 0}\left(I_{1}-3\right)^{i}+\sum_{i=1}^{N} \frac{1}{D_{i}}(J-1)^{2 i}, \\
& W=\sum_{i=1}^{N} \frac{2 \mu_{i}}{\alpha_{i}^{2}}\left(\lambda_{1}^{\alpha_{i}}+\lambda_{2}^{\alpha_{i}}+\lambda_{3}^{\alpha_{i}}-3\right)+\sum_{i=1}^{N} \frac{1}{D_{i}}(J-1)^{2 i}
\end{aligned}
$$

where: $\alpha_{i}$ and $\mu_{i}$ are material constants.

$$
W=\mu\left\{-\left(\lambda_{m}^{2}-3\right)[\ln (1-\eta)+\eta]-\frac{2}{3} \alpha\left(\frac{I-3}{2}\right)^{\frac{3}{2}}\right\}+\frac{1}{D}\left(\frac{J^{2}-1}{2}-\ln J\right)
$$

where

$$
\begin{aligned}
& I=(1-\beta)\left(\lambda_{1}^{2}+\lambda_{2}^{2}+\lambda_{3}^{2}\right)+\beta\left(\frac{1}{\lambda_{1}^{2}}+\frac{1}{\lambda_{2}^{2}}+\frac{1}{\lambda_{3}^{2}}\right), \\
& \eta=\sqrt{\frac{I-3}{\lambda_{m}^{2}-3}},
\end{aligned}
$$

$\mu$ - initial shear modulus,

$\lambda_{m}$ - locking stretch,

$\alpha$ - global interaction parameter,

$\beta$ - linear mixture parameter.

The Marlow model was also tested. This model does not contain any explicit relation between the strain energy density and compressibility. It is assumed that the strain energy density is only a function of the first invariant of the strain tensor and for incompressible materials $\left(I_{3}=0\right)$. It can be written as Eq.(4.8).

$$
W=\lambda_{I}^{2}+\lambda_{2}^{2}+\frac{1}{\lambda_{I}^{2} \cdot \lambda_{2}^{2}} .
$$




\section{Research methods}

On the basis of the characteristics of uniaxial tension, constant coefficients present in the expressions of the strain energy models were identified. It was limited to a maximum of fifth-order polynomials. A standard tool implemented in the Abaqus system, designed to the fit polynomial coefficients, which were obtained on the basis of test data [14], was used for this purpose. Because the results came from uniaxial tests, it was assumed that there are relations (5.1) between appropriate stretches and stretches in the direction of load action $\lambda_{U}$.

$$
\begin{aligned}
& \lambda_{1}=\lambda_{U}, \\
& \lambda_{2}=\lambda_{3}=\frac{1}{\sqrt{\lambda_{U}}}
\end{aligned}
$$

where

$$
\lambda_{U}=\varepsilon_{U}+1,
$$

$\varepsilon_{U}-$ nominal strain in the stretching direction.

Stability analyses of identified models were conducted using Drucker's criteria [15, 14]. On this basis it can be concluded that the models which fulfil these criteria are generally well suited for numerical analysis, and those that do not, can cause problems with obtaining solution convergence or getting the correct result. These criteria make it possible to evaluate the extent of strains for which the identified model can work properly, and beyond which we should expect problems. Expecting that for one material tested Drucker's criteria may be fulfilled by several models, it was assumed the smallest value of the stress average deviation of the characteristic resulting in the identification process, compared to actual characteristic, as a criteria of fitting material models.

The last stage of the evaluation of identified material models is an assessment of the results of convergence obtained from numerical analyses, conducted using the finite element method (FEM), with the results obtained with the actual tensile tests. It is a necessary condition, notwithstanding the fulfillment of Drucker's criteria. The FEM analyses were performed using the Abaqus system, ver.6.13. The FEM model was made in accordance with the dimensions presented in Fig.2 as a three-dimensional model shown in Fig.3. It was constructed from $172 \mathrm{C} 3 \mathrm{D} 8 \mathrm{RH}$ elements which are available in the ABAQUS/Standard library (C3D8RH 8-node brick linear, reduced integration with hourglass control, hybrid elements). It used the object symmetry. The implementation of boundary conditions and loads is shown in Fig.4.

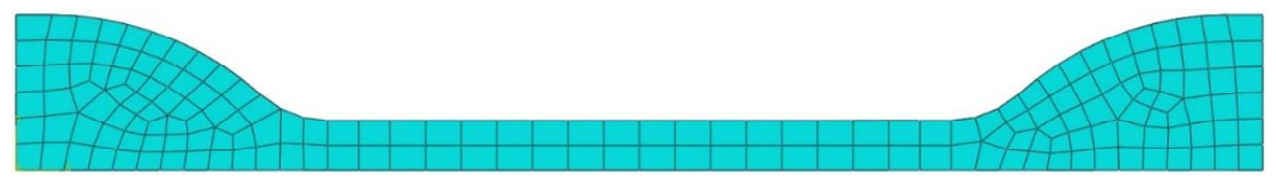

Fig.3. FEM mesh. 


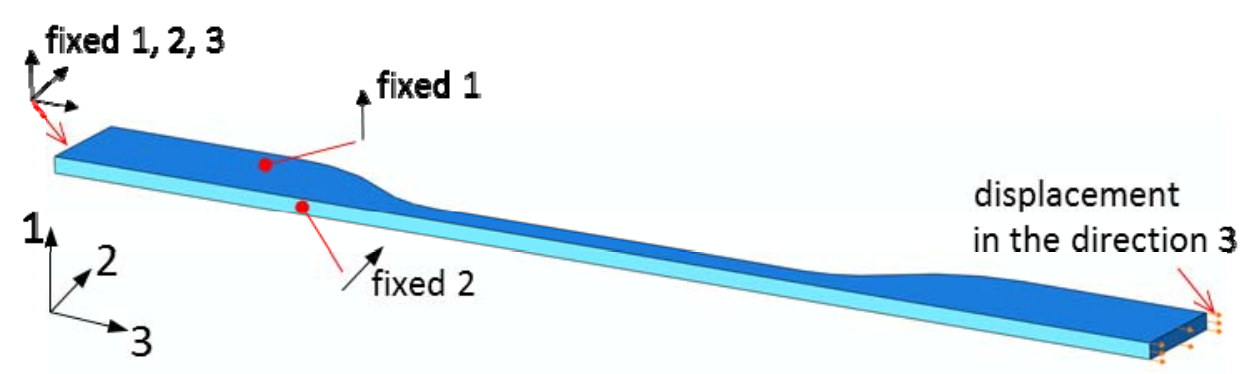

Fig.4. Boundary conditions and the way of load realization.

\section{Results}

Based on the analysis of uniaxial tensile data of tested digital materials, their ultimate tensile strength $(\mathrm{Rm})$ was set. The results are shown in Tab.1. The range of data included in the process of identification of factors of tested material models was limited to Rm.

Table 1. Tensile strength of the materials from the Tango and Vero groups.

\begin{tabular}{|l|l|l|l|}
\hline Tango series & $\begin{array}{l}\text { ultimate tensile strength } \\
{[\mathrm{MPa}]}\end{array}$ & \multicolumn{1}{|c|}{ Vero series } & $\begin{array}{l}\text { ultimate tensile strength } \\
{[\mathrm{MPa}]}\end{array}$ \\
\hline Tango95 & 4.40 & VeroWhite & 40.09 \\
\hline Tango85 & 3.15 & Vero20 & 42.12 \\
\hline Tango70 & 1.87 & Vero25 & 44.65 \\
\hline Tango60 & 1.07 & Vero35 & 43.65 \\
\hline Tango50 & 0.61 & Vero40 & 36.04 \\
\hline Tango40 & 0.59 & Vero50 & 32.11 \\
\hline TangoBlack & 0.39 & Vero60 & 24.12 \\
\hline
\end{tabular}

\subsection{Materials from Tango group}

Figure 5 shows the results of strain energy identification of models fulfilling Drucker's criteria, in the whole range of tested strains for each tested material from the Tango group. On the basis of the adopted criteria of fitting material models, the best models were determined. The results are shown in Tab.2. 

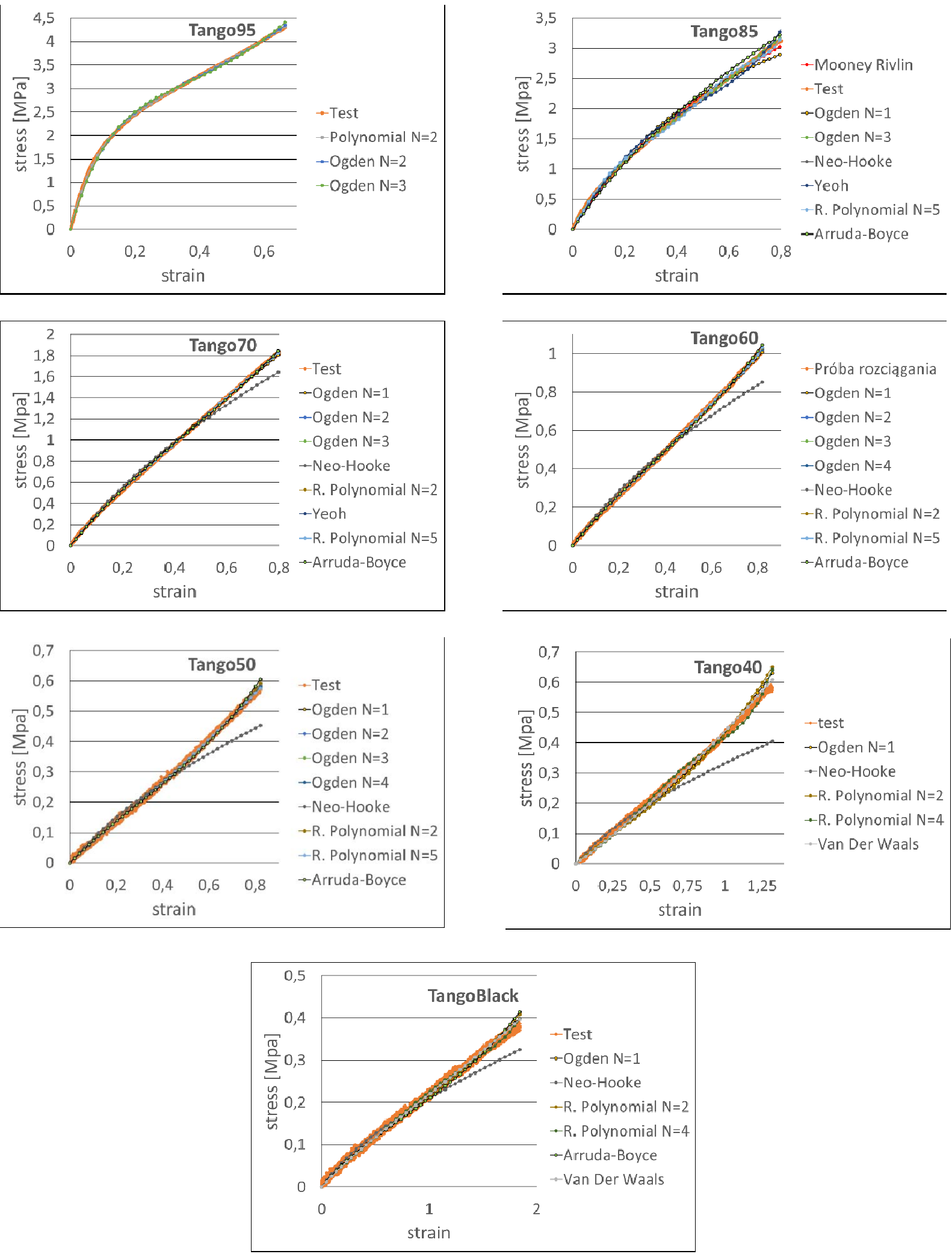

Fig.5. Models from Tango group fulfiling the Drucker's criteria in whole range of tested strains (to $\mathrm{Rm}$ ). 
The results presented in Tab.2 were used in the FEM models. A comparison of the FEM analysis results with the actual tensile tests results from the Tango group materials is shown in Fig.6.

Table 2. Summary of the best strain energy models of the Tango group materials.

\begin{tabular}{|c|c|c|c|c|c|c|c|c|c|c|}
\hline \multirow[t]{3}{*}{ Material } & \multicolumn{10}{|c|}{ strain energy models and its factors } \\
\hline & \multicolumn{10}{|c|}{ Reduced Polynomial $N=5$} \\
\hline & $\bar{i}$ & $D$ & \multicolumn{2}{|l|}{$C_{i 0}$} & \multicolumn{2}{|l|}{$C_{i 1}$} & $C_{i 2}$ & $C_{i 3}$ & $C_{i 4}$ & $C_{i 5}$ \\
\hline \multirow{5}{*}{ Tango95 } & 1 & 0 & 3.628636360 & & 0 & & $\overline{0}$ & 0 & 0 & 0 \\
\hline & 2 & 0 & -7.017715580 & & 0 & & 0 & 0 & & \\
\hline & 3 & 0 & 13.279605400 & & 0 & & 0 & & & \\
\hline & 4 & 0 & -12.166401500 & & 0 & & & & & \\
\hline & 5 & 0 & 4.221144020 & & 0 & & & & & \\
\hline \multirow{7}{*}{ Tango 70} & 1 & 0 & 0.5273158620 & & 0 & & 0 & 0 & 0 & 0 \\
\hline & 2 & 0 & -0.0030200197 & & 0 & & 0 & 0 & & \\
\hline & 3 & 0 & 0.1228237480 & & 0 & & 0 & & & \\
\hline & 4 & 0 & -0.0085804592 & & 0 & & & & & \\
\hline & 5 & 0 & 0.0020487938 & & 0 & & & & & \\
\hline & \multicolumn{10}{|c|}{$\operatorname{Ogden} N=3$} \\
\hline & $i$ & 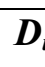 & \multicolumn{3}{|l|}{$\mu_{i}$} & \multicolumn{5}{|c|}{$\alpha_{i}$} \\
\hline \multirow{3}{*}{ Tango85 } & 1 & 0 & \multicolumn{3}{|l|}{-21.66975410} & \multicolumn{5}{|c|}{-1.96012445} \\
\hline & 2 & 0 & \multicolumn{3}{|l|}{7.63365831} & \multicolumn{5}{|c|}{-0.74423550} \\
\hline & 3 & 0 & \multicolumn{3}{|l|}{17.05402920} & \multicolumn{5}{|c|}{-3.32434835} \\
\hline \multirow{5}{*}{ Tango60 } & 1 & 0 & \multicolumn{3}{|l|}{-1.62330062} & \multicolumn{5}{|c|}{0.00156382} \\
\hline & 2 & 0 & \multicolumn{3}{|l|}{1.38837179} & \multicolumn{5}{|c|}{1.76299002} \\
\hline & 3 & 0 & \multicolumn{3}{|l|}{0.76364831} & \multicolumn{5}{|c|}{-3.37039362} \\
\hline & & $\bar{n} 1$ & & & & & & & & \\
\hline & $i$ & $D$ & $\mu_{i}$ & & & & & $\alpha_{i}$ & & \\
\hline & 1 & 0 & 0.28254910 & & & 2.0 & 5863 & & & \\
\hline & 2 & 0 & 0.00703673 & & & 3.9 & 5197 & & & \\
\hline 1 angos0 & 3 & 0 & -0.48776631 & & & -1.5 & 95760 & & & \\
\hline & 4 & 0 & 0.39556415 & & & & 95208 & & & \\
\hline & & ler & als & & & & & & & \\
\hline & $D$ & & $\mu$ & & $\lambda_{n}$ & & & $a$ & & $\beta$ \\
\hline Tango40 & $\overline{0}$ & & 64341 & & .613 & & & 053405 & & 0 \\
\hline TangoBlack & 0 & & 51330 & & 9176 & & & 304595 & & 0 \\
\hline
\end{tabular}




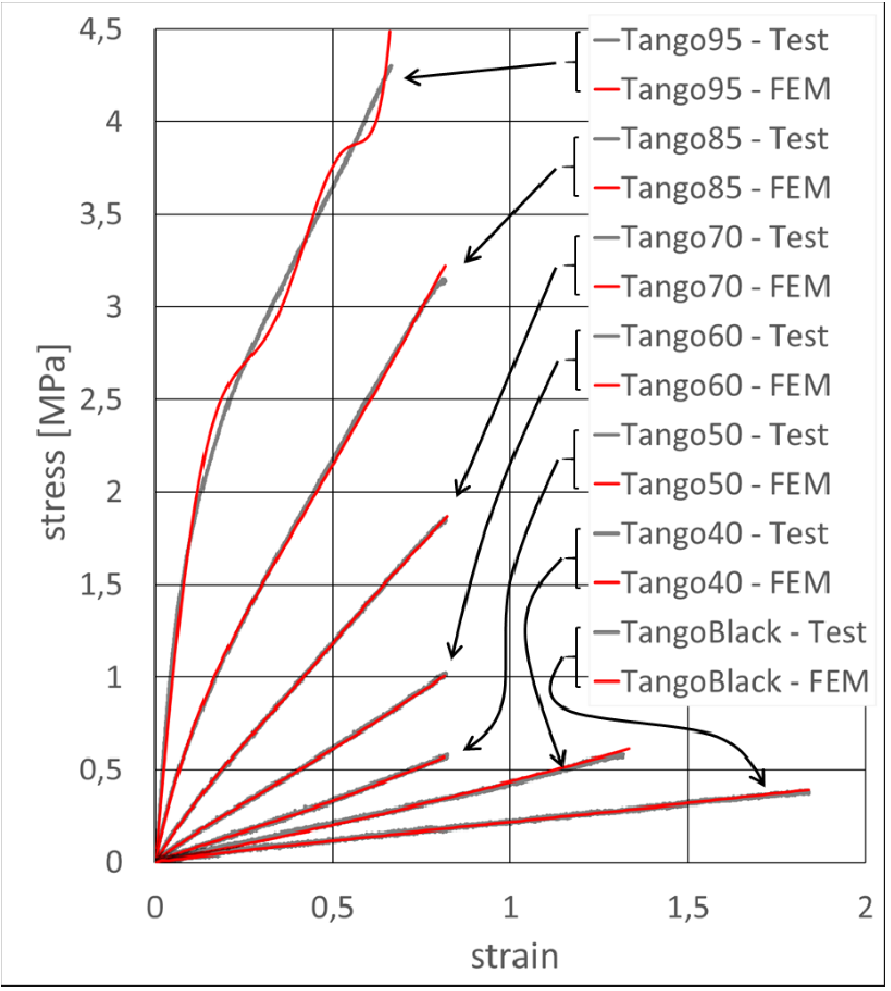

Fig.6. Comparison of the FEM analysis results with tensile tests results from the Tango group materials.

\subsection{Materials from Vero group}

Figure 7 shows the results of strain energy identification of models fulfilling Drucker's criteria, in the whole range of tested strains for each tested material from the Vero group. For all tested materials from the Vero group the best fitting results were obtained for the Marlow model. A comparison of the FEM analysis results with actual tensile tests results from the Vero group materials is shown in Fig.8. 

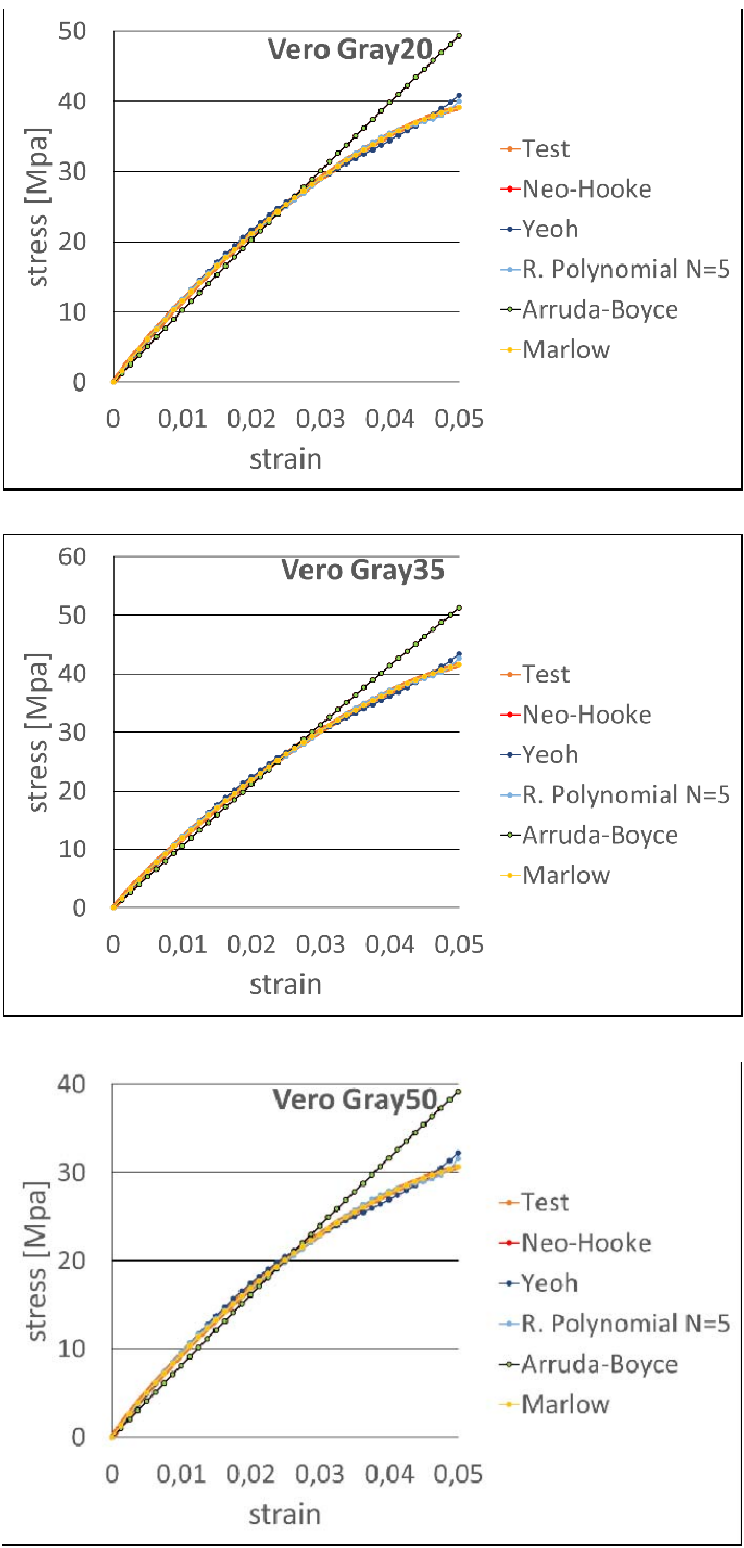
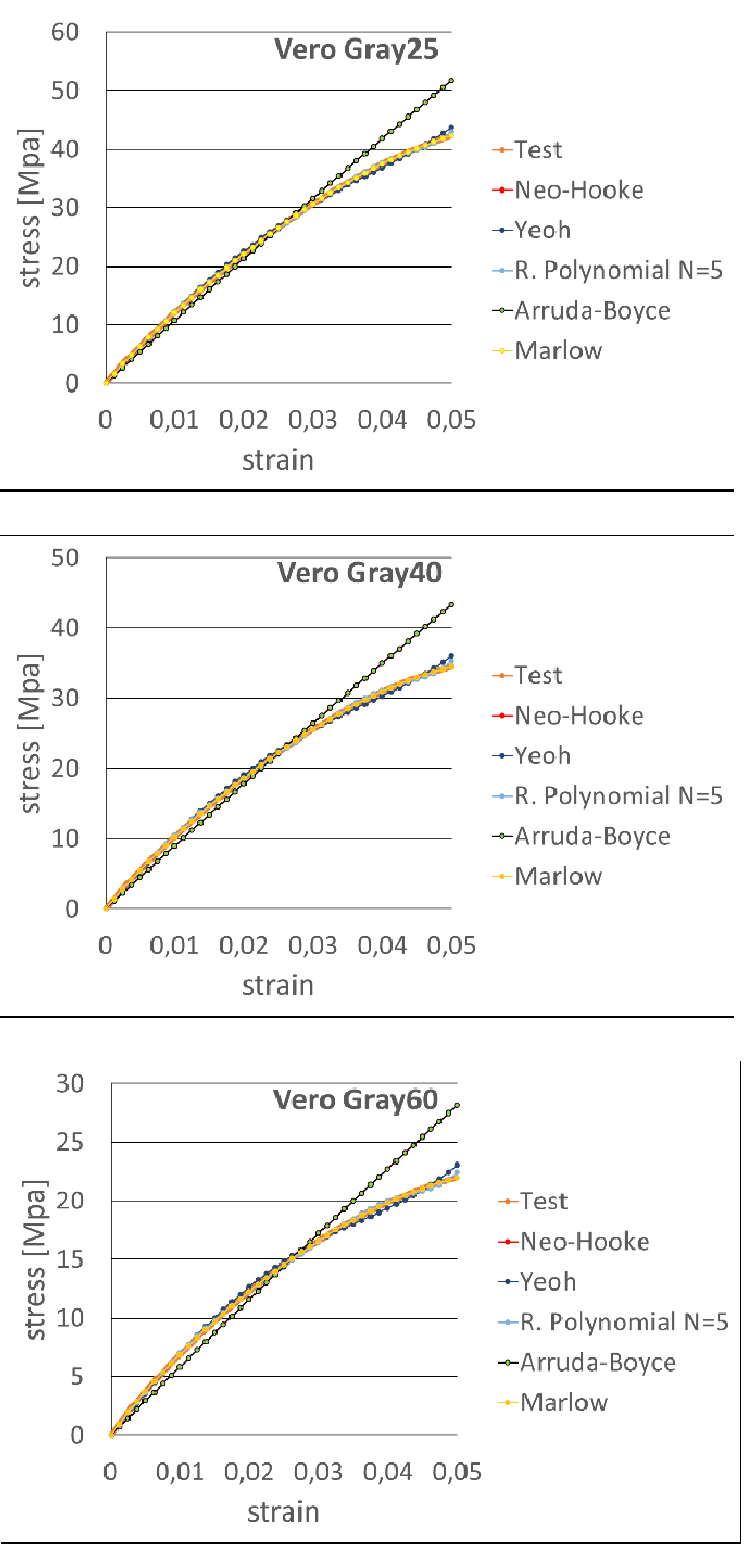

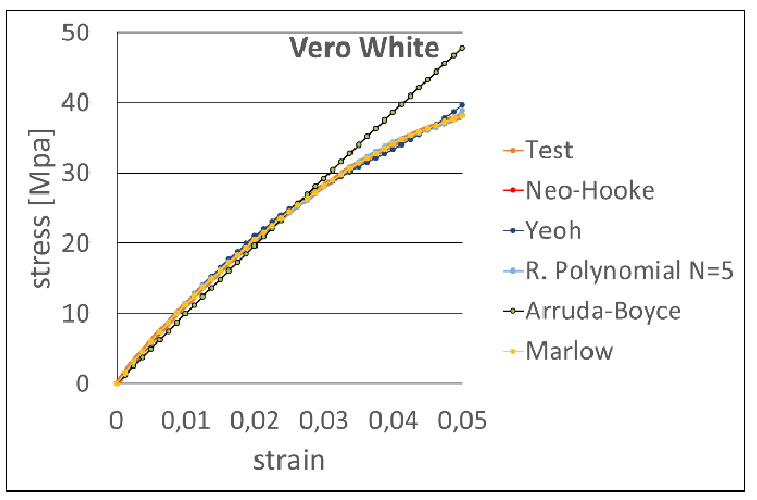

Fig.7. Models from Vero group fulfiling the Drucker's criteria in whole range of tested strains (to $R m$ ). 


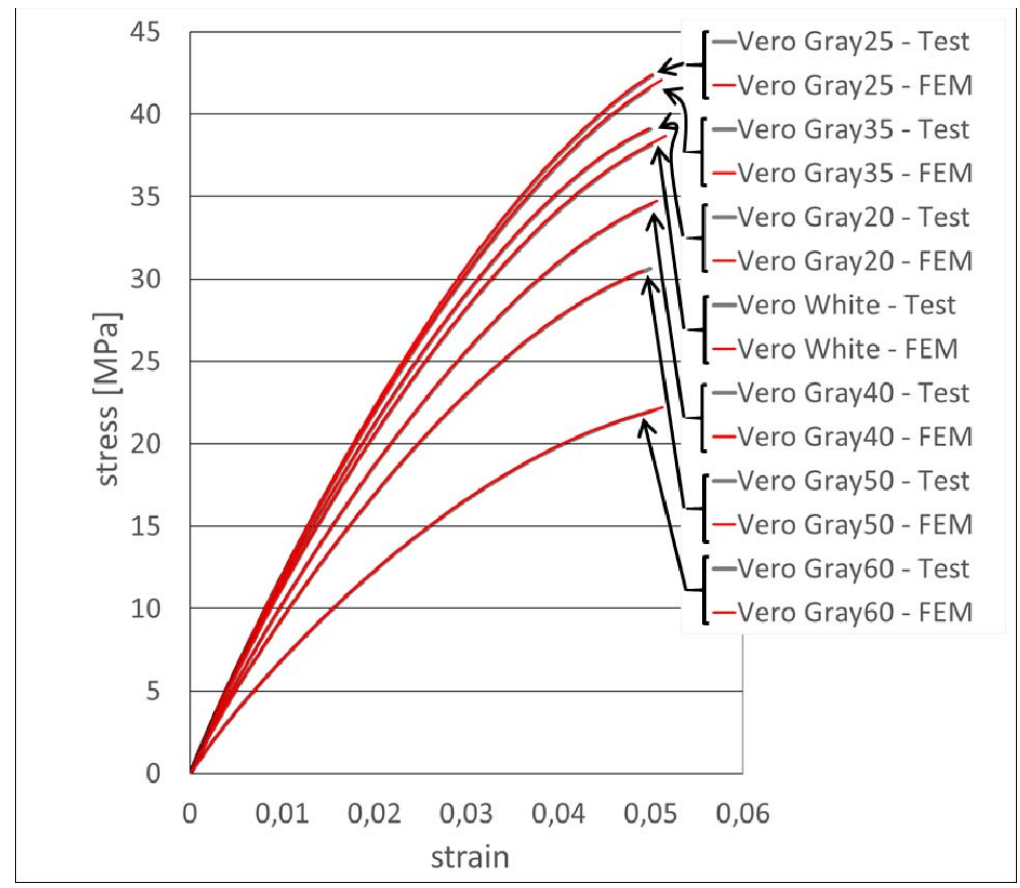

Fig.8. Comparison of the FEM analysis results with tensile tests results from the Vero group materials.

\section{Conclusions}

The aim of the study was the evaluation of the possibility of using selected hyperelastic materials models for the description of constitutive equations of digital materials. The aim was attained by performing numerical analysis and examination of uniaxial stretching of fourteen different materials characteristics, divided into two groups:

- $\quad$ Tango - very flexible materials having an ultimate tensile strength from 0.39 to $4.40 \mathrm{MPa}$.

- Vero - far stiffer materials with an ultimate tensile strength from 24.12 to $44.65 \mathrm{MPa}$.

\section{Nomenclature}

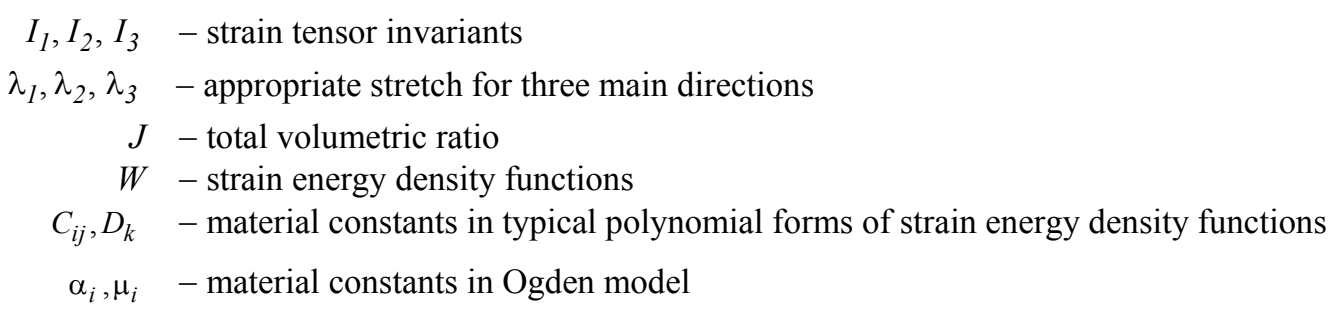

\section{References}

[1] Popescu G. (2007): Digital Materials for Digital Fabrication. - http://cba.mit.edu/ docs/theses/07.08.Popescu.pdf, (acces 02.02.2016).

[2] Siemiński P. and Budzik G. (2015): Additive manufacturing technologies: 3D printing, 3D printers. - Warsaw: Publishing House of the Warsaw University of Technology.

[3] Stratasys. (2015): PolyJet Technology. - http://www.stratasys.com/3d-printers/technologies/polyjet-technology, (acces 10.20.2015). 
[4] Zhou C., Chen Y., Yang Z. and Behrokh K. (2011): Development of a Multi-material Mask-Image-Projectionbased Stereolithography for the Fabrication of Digital Materials. - In: Solid Freeform Fabrication Symposium 2011, https://sffsymposium.engr.utexas.edu/Manuscripts/2011/2011-06-Zhou.pdf, (12.05.2016).

[5] Hiller J. and Lipson H. (2009): Design and analysis of digital materials for physical 3D voxel printing. - Rapid Prototyping Journal, vol.15, pp.137-149.

[6] Reichl K. and Inman D. (2016): Dynamic modulus properties of Objet Connex 3D Printer digital materials. - In: Topics in Modal Analysis \& Testing, Volume 10: Proceedings of the 34th IMAC, A Conference and Exposition on Structural Dynamics 2016 (M. Mains).

[7] Łąpieś Z., Żrodowski Ł. and Siemiński P. (2015): Mechanical And Microstructural Analysis of PolyJet Matrix Digital Materials. - In: Proceedings of the International Conference "Methods \& Tools for CAE - Concepts and Applications". - Bochnia: Warsaw University of Technology.

[8] Stratasys. (2015): PolyJet Materials Data Sheet. - http://usglobalimages.

stratasys.com/Main/Files/Material_Spec_Sheets/MSS_PJ_PJMaterialsDataSheet.pdf?v=635785205440671440, (acces 10.20.2015).

[9] EN ISO 527-2. (1996): Determination of tensile properties of plastics.

[10] Boyce M. and Arruda E. (1993): A three-dimensional constitutive model for the large stretch behawior of rubber elastic materials. - Journal of the Mechanics and Physics of Solids, pp.389-412.

[11] Guo Z. and Shuys L. (2008): Constitutive modeling of hyperelastic rubber-like materials. - Heron, (53), pp.109132.

[12] Chandrasekharaiah D. and Debnath L. (1994): Continuum Mechanics. - London: Academic Press Limited.

[13] Boyce M. and Arruda E. (2000): Constitutive models of rubber elasticity: a review. - Rubber Chemistry and Technology, (73), pp.504-523.

[14] Dassault Systèmes. (2013): Abaqus Analysis User's Guide.

[15] Jemioło S. (2002): Study of hyperelastic properties of isotropic materials, modeling and numerical implementation (Vol. "Building" No.140). - Warsaw: Publishing House of the Warsaw University of Technology.

Received: March 16, 2017

Revised: July 3, 2017 